\title{
Management of Comminuted Subtrochanteric Femur Fractures by Indirect Reduction and Biological Fixation with Dynamic Condylar Screw
}

\section{Mohamed Mansour Elzohairy*}

Lecturer and Consultant of Orthopaedic Surgery, Faculty of Medicine, Zagazig University, Egypt

Clinical Orthopaedic Fellow, Toronto University, Canada

\begin{abstract}
Background: The Comminuted subtrochanteric femoral fractures are considered as one of the most difficult fractures to treat in the orthopaedic literatures. The aim of this prospective study was to evaluate the indirect reduction and biological fixation technique with Dynamic condylar screw and plate fixation for the treatment of comminuted subtrochanteric femoral fractures.

Method: Thirty one cases suffered from comminuted subtrochanteric fractures femur, AO classification (type C), were treated by biological fixation, indirect reduction method with Dynamic condylar screw and plate fixation.

Results: The cases were followed up by clinical and radiological examination from 12 to 32 months (mean 22 months). In all cases union in acceptable alignment were achieved from 15 to 24 weeks (range from 3 to 6 months) (mean 4.5 months) in all cases except, three cases $(9.7 \%$ ) with failures of the fixations due to osteoporosis and early full weight bearing one week after surgery. With exception of 3 cases with fixation failures who excluded from the analysis of the functional results, all the cases had good functional outcome as regarding to Harris hip score which varying from 70 to 95 (mean 85).

Conclusion: Indirect reduction and biological fixation with Dynamic condylar screw and plate fixation is considered as a valuable cheap fixation method for the management of comminuted subtrochanteric fractures femur especially in young patients' populations.
\end{abstract}

Keywords: Comminuted; Subtrocahnteric femur; Fracture; Biological fixation; Dynamic condylar screw

\section{Introduction}

Comminuted subtrochanteric femoral fractures are considered as one of the most difficult fracture to treat for the orthopaedic surgeons $[1,2,3,4]$. Until now there is no agreement in the orthopaedic literatures for the best method to manage such complicated fractures $[2,4,5]$. The biological fixation is considered now as one of the golden solutions for this complicated fracture. The aim of biological fixation is not to cause any biological harm to the bone and the surrounding soft tissues by indirect reduction, proper alignment of the intermediate bone fragment $[1,6]$. The risks of open reduction includes, devitalization, damages of the vascular supply, soft tissues and increased risks of non union, infection and implant failure $[1,6]$. The aim of this study was to evaluate the results of comminuted subtrochanteric femoral fractures treated by indirect reduction and biological fixation method with Dynamic condylar screw and plate fixation.

All the patients gave the informed consent prior to being included in this study; the study was authorized by the local ethical committee and was performed in accordance with the Ethical standards of the 1964 Declaration of Helsinki as revised in 2000.

\section{Patients and methods}

From the beginning of August 2005 to October 2009, thirty one cases suffered from comminuted subtrochanteric fractures femur, $\mathrm{AO}$ classification (type C), were managed at our university trauma hospital, by biological fixation, indirect reduction method with Dynamic condylar screw and plate. They were 21 males and 10 females their age varying from 37 years to 70 years (mean 53.5) the causes of trauma were car accidents in all the cases. Right side fractures were in 18 cases and left side fractures were in 13 cases. All the fractures were closed, three cases were multiple trauma patients, one had stable fractures left third and fourth ribs who treated conservatively, the second had fractures right both bones forearm who treated by ORIF with small DCP and the third case had fractures right third and fourth metacarpals who treated by ORIF with kirschner wires fixation. AO Classification was used for the fractures classifications. Harris hip score was used for the clinical evaluation of the cases. The deformities were evaluated in the three planes according to coronal plane (varus or valgus), sagittal plane (anterior or posterior angulations and transverse plane external and internal rotation deformities and shortening.

\section{Operative technique}

The operations were done from the second to the fourth post trauma days. General anesthesia was used in 11 cases and spinal anesthesia was used in 20 cases. Orthopaedic table with traction and supine position was used in all the cases under image intensifier. Two incisions were used the upper one varying from 5 to $7 \mathrm{~cm}$ over the greater trochanter the second incision varying from 6 to $8 \mathrm{~cm}$ for the distal fixation below the comminuted fractures. Guide pin inserted first making angle $95^{\circ}$ $\mathrm{C}$ with the distal femur, followed by lag screw insertion over the guide pin then followed by subvastus lateralis extraperiosteal condylar plate

*Corresponding author: Mohamed Mansour Elzohairy, Lecturer and Consultant of Orthopaedic Surgery, Faculty of Medicine, Mansour Elzohairy Villa, 4 Zeid Ben Sabet Street, University Villas, Zagazig, Sharkia Governorate, Egypt, Tel: +201124467856; E-mail: elzohairy2020@yahoo.com

Received March 12, 2012; Accepted May 20, 2012; Published May 25, 2012

Citation: Elzohairy MM (2012) Management of Comminuted Subtrochanteric Femur Fractures by Indirect Reduction and Biological Fixation with Dynamic Condylar Screw. J Trauma Treat 1:136. doi:10.4172/2167-1222.1000136

Copyright: (C) 2012 Elzohairy MM. This is an open-access article distributed unde the terms of the Creative Commons Attribution License, which permits unrestricted use, distribution, and reproduction in any medium, provided the original author and source are credited. 
insertion from proximal to distal with the barrel directed toward outside, then the barrel rotated $180^{\circ} \mathrm{C}$ by the aid of screw driver and inserted over the lag screw. From four to six cortical screws fixed distally below the fracture site. Then the traction released and the compression screw tightened to lag screw to make stable construct with the plate and the lag screw. Wound closed in layers and suction drains were applied for both wounds. Drains were removed 48 hours post operative and sutures were removed 14 days post operative.

\section{Post operative rehabilitation}

Exercise in bed was started from the second post operative day. Non weight bearing was started from the third to the fourth post operative weeks then was followed by partial weight bearing after three to four weeks according to the factures configurations, bone quality and the stability of the fractures fixation. Full weight bearing was started when the fractures showed complete union clinically by absence of limb pain when standing upon the fractured limb alone and radiologically by the presence of the abundant callus at least in two views.

\section{Results}

The cases were followed up by clinical and radiological examination from 12 to 32 months (mean 22 months), (Figure 1a, 1b, and 1c) and the deformities were evaluated in the three planes, the coronal plane (varus or valgus), the sagittal plane (anterior or posterior angulations) and the transverse plane external and internal rotation deformities and shortening. In all the cases union were achieved from 15 to 24 weeks (range from 3 to 6 months) (mean 4.5 months) with Acceptable alignment (less than $10^{\circ}$ varus/valgus or anterior/posterior angulations and rotation) except, three cases (9.7\%) with failures of the fixations due to osteoporosis and early full weight bearing one week after surgery. One with cut through of the lag screw failure, who converted to cemented total hip arthroplasty (3.2\%), the second with loss of the fixation who need revision by proximal femoral nail (3.2\%), and the third case with bending of the plate with coxa vara and persistent limp who refused further surgery and was satisfied with the results (Figure $2 \mathrm{a}$ and $2 \mathrm{~b}$ ).

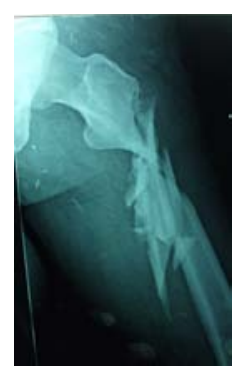

a
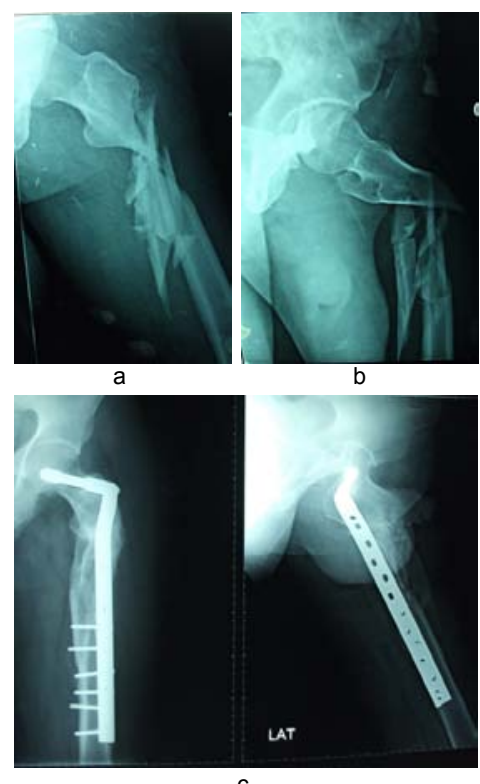

Figure 1: (a) and (b) 42 years old male with comminuted subtrochanteric fracture (c) the last follow-up with union in Acceptable alignment 32 months post operative.
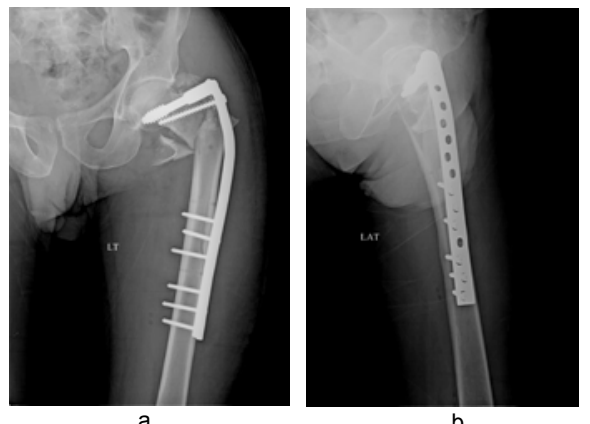

Figure 2: 70 years old male with comminuted subtrochanteric fracture with bending of the plate 1 month post operation due too early full weight bearing 1 week after operation.

\begin{tabular}{|l|c|}
\hline Complications & Percentages \\
\hline 1.cut through & One case $(3.2 \%)$ \\
\hline 2.plate bending & One case $(3.2 \%)$ \\
\hline 3.loss of fixation & One case $(3.2 \%)$ \\
\hline 4.superficial wound infection & Two cases $(6.5 \%)$ \\
\hline
\end{tabular}

Table 1: Complications and percentages.

Shortenings occurred in 6 cases, $1.5 \mathrm{~cm}$ occurred in 4 cases $(12.9 \%)$ and $2.5 \mathrm{~cm}$ occurred in 2 cases $(6.5 \%)$ which were acceptable and adaptable by the patients with shoe lifts. Superficial infection occurred in two cases $(6.5 \%)$ that treated by repeated dressings, IV antibiotic for one week and oral antibiotic for another week. With exception the 3 cases with fixation failures who excluded from the analysis of the functional results, all the other cases had good functional outcome as regarding to Harris hip score which varying from 70 to 95 (mean 85). Complications are listed in (Table 1).

\section{Discussion}

Open anatomical reduction with or without valgus medial displacement osteotomy was the ideal method for that complicated unstable fractures, this concept was changed after the successful results of indirect reduction with biological fixation method $[1,2,3,4,5,6,7,8,9]$. The complex nature of this fractures type especially with the complex factures configurations that extended to the intertrochanteric fossa, makes this fractures very difficult to stabilize by interlocking nail [3]. The dynamic condylar screw and plate, working as a tensile plate which allows the indirect reduction of the facture fragments with buttressing of the comminuted lateral cortex [2]. There are many debates as regarding using either extramedullary or intramedullary fixations for those complicated fractures. Intramedullary fixation is biomechanically superior to extramedullary fixation. The functional evaluation showed no significant difference in pain, range of movement, or walking ability, but recovery was significantly earlier in patients with the Gamma nail than the Dynamic condylar screw and plate. Good results were reported in elderly patients after intramedullary fixation. Indirect reduction and mini-incision plating is a reliable alternative in subtrochanteric fractures even in the elderly. Malunion was reported in a patient with $\mathrm{AO}$ type $\mathrm{C}$ fracture due to severe comminution. Malunion (coxa vara) was reported in $6 \%$ of the patients and was painless and functionally acceptable, with no obvious restriction of hip movement or deformity. Malunion may not be symptomatic and require further treatment. A mean shortening of 1.2 to $1.5 \mathrm{~cm}$ occurred in 27 to $42 \%$ of patients; none of them was limping. Intramedullary devices require less surgical exposure, enable early weight bearing, achieve better proximal 
Citation: Elzohairy MM (2012) Management of Comminuted Subtrochanteric Femur Fractures by Indirect Reduction and Biological Fixation with Dynamic Condylar Screw. J Trauma Treat 1:136. doi:10.4172/2167-1222.1000136

Page 3 of 3

fixation, and exert less biomechanical stresses (as the lever arm is moved medially) [6]. However, they are not suitable for subtrochanteric fractures with intertrochanteric extension and are associated with technical difficulties in up to $63 \%$ of cases. Reconstruction nailing is technically demanding; plate and screw fixation is probably the best option. Indirect reduction and condylar blade plate (СBP) fixation achieved excellent results in comminuted subtrochanteric fractures, despite being technically demanding. Sliding hip screws (DHS) are technically straight forward, but anchoring the proximal fragment cannot be supplemented with screws [6]. Dynamic condylar screws (DCS) simplify fixation and require less-exacting technique than CBPs. Dynamic condylar screws insertion requires a high level of skill and although the intramedullary fixation is theoretically biomechanically stronger and less invasive on the clinical ground, however no significant difference where found in the comparative studies between the two methods in all the series $[6,7,10,11]$. There is also other advantage of Dynamic condylar screws that, it is cheaper than the intramedullary nail especially in developing countries [6]. Compared to the condylar blade plate (CBP), the dynamic condylar screw and plate (DCS) fixation is easier and simpler in the fixation as it requires only two plane alignments while the condylar blade plate (CBP) requires three plane alignments, with this difficulty present especially in the people with narrow antero-posterior diameter of the femoral neck with wide (CBP) blade $[6,8]$. As regarding the age selection for this fixation method, Kulkarni and Moran [5] in their series found that the dynamic condylar screw and plate (DCS) is an effective implant in young patients with high- energy injuries who were able to remain partial or non weight bearing during their rehabilitation, however they do not recommend the dynamic condylar screw and plate (DCS) for the elderly population with low- energy injuries because the high failure rate due to difficult restriction of weight bearing in this group of patients with early weight bearing. In our study all the fixations failures occurred in elderly group of patients from 65 years to 70 years who represent $(9,7 \%)$ of all our cases because it were difficult for them to remain partial or non weight bearing during their rehabilitation. This considered as limitation in our study because we did not had enough numbers of elderly group of patients to compare. Recently there were successful reports of the biomechanical and the clinical studies trails $[11,12]$ of the trochanteric fixation nail using a helical blade device for the fixation of unstable intertrochanteric hip fractures commonly seen in the elderly patients with osteoporosis, in the future this method will be investigated by us for this group of patients.

\section{Conclusions}

Based on our results, we found that indirect reduction and biological fixation method with the dynamic condylar screw and plate (DCS) is considered as a valuable cheap fixation method for the management of comminuted subtrochanteric fractures femur especially in the young patient's populations.

\section{References}

1. Miclau T, Martin ER (1997) The evolution of modern plate Osteosynthesis. Injury 28: A3-A6.

2. Celebi L, Can M, Murati HH, Yagmurlu MF, Yuksel HY, et al. (2006) Indirect reduction and Biological internal fixation of comminuted subtrochanteric fractures of the femur. Injury 37: 740-750.

3. Sienbenrock AK, Müller U, Ganz R (1998) Indirect reduction with a condylar blade plate for osteosynthesis of subtrochanteric femoral fractures. Injury 29 : C7-C15.

4. Nikolaou VS, Papathanasopoulos A, Giannoudis PV (2008) What's new in the management of proximal femoral fractures? Injury 39: 1309-1318.

5. Kulkarni SS, Moran CG (2003) Results of dynamic condylar screw for subtrochanteric fractures. Injury 34: 117-122.

6. Rohilla R, Singh R, Magu KN, Sangwan SS, Devgun A, et al. (2009) Technical aspects of the use of dynamic condylar screw in biological fixation of comminuted subtrochanteric fractures. Eur J Orthop Surg Traumatol 19: 33-37.

7. Krettek C, Schandelmaier P, Miclau T, Tscheme H (1997) Minimally invasive percutaneous plate osteosynthesis (MIPPO) using the DCS in proximal and distal femoral fractures. Injury 28: A20-A30.

8. Vaidya SV, Dholakia DB, Chatterjee A (2003) The use of a dynamic condylar screw and biological reduction techniques for subtrochanteric femur fracture. Injury 34: 123-128.

9. Kesemenli C, Subasi M, Necmioglu S, Kapukaya A (2002) Treatment of multifragmentary fractures of the femur by indirect reduction (biological) and plate fixation. Injury 33: 691-699.

10. Schipper IB, Marti RK, Van der Werken C (2004) Unstable trochanteric femora fractures: extramedullary or intramedullary fixation. Review of literature. Injury 35: $142-151$

11. Strauss E, Frank J, Lee J, Kummer FJ, Tejwani N (2006) Helical blade versus sliding hip screw for treatment of unstable intertrochanteric hip fractures: a biomechanical evaluation. Injury 37: 984-989.

12. Gardner MJ, Briggs SM, Kopjar B, Helfet DL, Lorich DG (2007) Radiographic outcomes of intertrochanteric hip fractures treated with the trochanteric fixation nail. Injury 38: 1189-1196. 